\title{
Skeletal Muscle Atrophy in Chronic Kidney Disease (CKD)
}

\author{
Javier Perez ${ }^{1}$, Sharon $\mathrm{Moe}^{2}$, Neal Chen², Keith Avin ${ }^{3}$ \\ ${ }^{1}$ Indiana School of Medicine, ${ }^{2}$ Indiana School of Medicine, Department of Nephrology, ${ }^{3}$ Indiana \\ University, Department of Physical Therapy
}

\begin{abstract}
Background:
Skeletal muscle atrophy and dysfunction occur with chronic kidney disease (CKD) progression leading to morbidity, mortality, and falls. Skeletal muscle dysfunction may be due to impaired fatty acid (FA) oxidation and enhanced pyruvate oxidation as demonstrated in preliminary metabolomic data. We performed multiple techniques to assess the extent of muscle dysfunction and associated pathways, including: systematic review and meta-analysis, assays of disease progression and FA metabolism, expression of markers associated with skeletal muscle FA metabolism and pyruvate oxidation.
\end{abstract}

\section{Methods:}

Meta-analysis: Multiple databases were used to identify relevant studies of muscle atrophy in preclinical and clinical models.

Experimental Study: 1)CKD rats and 2)Normal littermates $(\mathrm{N}=12 / \mathrm{gr})$ at 35 weeks. Extensor digitorum longus (EDL) and soleus were harvested at sacrifice. Serum Biochemistry: Plasma BUN, calcium and phosphorus were analyzed using colorimetric assays. Carnitine Assay: Plasma carnitine levels was measured using ELISA kit. Protein Expression: Western blots with the lysate of EDL and soleus to determine the activity levels of PDH and PDK4.

\section{Results:}

A total of 4685 studies were screened in the meta-analysis, of which 646 were relevant. Subsequent steps are to perform full text review and data extraction. Animal studies: BUN and phosphorus were significantly increased in CKD compared to normal. Carnitine levels were significantly decreased in CKD rats compared to normal. PDH was not significantly different in the EDL or soleus. PDK4 is yet to be performed.

\section{Conclusions:}

The extent to which muscle atrophy occurs will be identified in the meta-analysis. Elevated BUN confirmed disease and carnitine assay confirmed low carnitine levels in CKD. Identifying low carnitine has led to an interventional study of carnitine supplementation to determine if there was improved FA oxidation. Testing of PDK4 is needed to determine significance of pyruvate regulation. Reviewing the literature and understanding the mechanism of skeletal muscle atrophy in CKD will allow future targeted therapeutics. 